\title{
Direct Torque Control in Hybrid System
}

\author{
Zhixue Tong, Rui Wang \\ The College of Mechanical Engineering, Xi'an University of Architecture and Technology
}

\begin{abstract}
Keywords: Hybrid; Direct torque control; Drive; Power Distribution.
\end{abstract}
\begin{abstract}
Hybrid system by two electric motors and power source to drive the vehicle engine is running, the two power sources in the vehicle running in how power affects the performance of the entire mixed distribution system. After the diesel engine because of its non-linear, multi-condition, time-varying delay and other characteristics, it is difficult to achieve real-time control, dual-axis parallel to the paper self-developed hybrid test bench for the study, the system switches to the individually driven by diesel hybrid drive, according to coupling torque values and the value of the motor torque output is set after the coupling between a hybrid system utilizing a hybrid drive system, torque, resulting in the relevant operating parameters of the diesel engine, to lay a foundation for a diesel engine control.
\end{abstract}

\section{A tested structure introduced}

\subsection{Test bench is introduced}

Hybrid experiment platform is designed based on the working principle of the hybrid mechanical and electrical integration of liquid experimental platform, experimental platform with motor and diesel engines as the main source of power, under the condition of stable load, motor as the main control objects, the direct torque control for motor control, so how to adjust the motor and the distribution of the diesel engine power output become the focus of the study.

Hybrid vehicles equipped with motor and engine, and other sources of power, in order to improve the energy conversion efficiency of the power system, may need to select a different combination between the power sources according to traffic, will form a different drive mode. Various working mode to normal work, coordination between hybrid system components are extremely complex, therefore, how to control the vehicle energy management and power system is the most important part of the hybrid solution, based on rules is still the current control strategy design of the main methods, in the hybrid electric vehicle research approach. The main problem to be solved is the control strategy torque distribution and battery charge state of the engine and the motor (SOC) control.

\subsection{Tested structure introduced}

Hybrid system structure of experiment platform is shown in figure 1, the test platform for biaxial parallel type hybrid system, the characteristics of this structure is clutch 1 responsible for diesel engine connected to the load, the clutch 2 implementation of motor and load connection. 1 and 2 of the clutch of the clutch and gear are placed in the closed box.

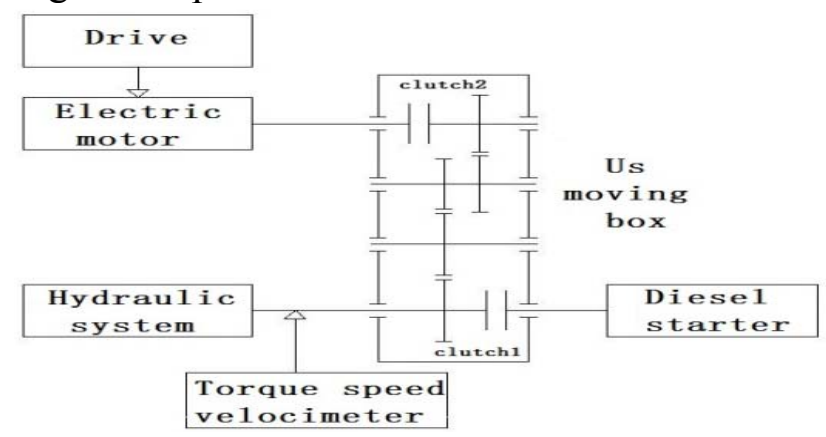

Figure 1 Parallel hybrid powertrain test platform

The main components of the hybrid system selection and parameters as shown in the table below, the hybrid system is a control system, the realization of the entire hybrid clutch connection and 
disconnection of control, the drive motor to achieve real-time control, done by PLC System power synthesis output.

Table 1. Hybrid system main components parameters

\begin{tabular}{cccll}
\hline Mode & $\begin{array}{c}\text { Engine } \\
\text { status }\end{array}$ & $\begin{array}{c}\text { Motor } \\
\text { Status }\end{array}$ & Clutch1 & Clutch2 \\
\hline Motor drive & Inoperation & Electric & Combine & Separate \\
\hline Engine driven & Operation & Inoperation & Separate & Combine \\
\hline Hybrid drive & Operation & Electric & Combine & Combine \\
\hline
\end{tabular}

\section{Control Strategy}

\subsection{The operation mode of the hybrid system}

Hybrid experiment platform based on the rules of the torque distribution of energy management system, Based on the steady state of engine characteristic figure, chose motor and engine direct torque control as the main control objects, when to meet the request of the power output, the reasonable assign torque reasonable to both of them.

Different drive mode for different load, diesel engine and electric motor through the clutch to achieve switching between different drive modes, this drive method, which can ensure power demand, and to ensure that significantly enhance the environmental effect of energy conservation and emissions reduction.

According to the function analysis of the experimental platform, the operation mode of the hybrid experiment platform can be determined, as following several kinds: pure electric drive mode, the engine drive model and hybrid model of the three model.

Table 2. Hybrid system working mode

\begin{tabular}{cccc}
\hline \multirow{2}{*}{ Part Name } & Mold & \multicolumn{2}{c}{ Main technical parameters } \\
\cline { 3 - 4 } Engine & \multirow{2}{*}{ Diesel starter } & Parameter & Numerical value \\
\cline { 3 - 4 } & \multirow{2}{*}{ Motor } & Asynchronous motor $/ \mathrm{kW}$ & $33 \mathrm{~kW}$ \\
\cline { 3 - 4 } & & Peak torque $/(\mathrm{N} \cdot \mathrm{m})$ & $160 \mathrm{~N} \cdot \mathrm{m}$ \\
\hline Transducer & MM440 & Peak torque $/(\mathrm{N} \cdot \mathrm{m})$ & $380 \mathrm{~V}$ \\
\hline
\end{tabular}

As can be seen from the table, the clutch engagement and disengagement can switch between different working conditions, in order to achieve the coordination between a pluralities of power sources.

\subsection{Clutch engagement torque control strategy before and after}

In this paper, the device is a parallel type hybrid experiment platform, analyze the different work mode of components, hybrid clutch combination can be attributed as follows: through the coordination of motor and the proportion of diesel engine output torque to control the entire system, namely the clutch driven by diesel engine alone before 2 joint load, the clutch 2 together, because of using motor direct torque control speed is decided by the speed of the load size, under the control of the frequency converter, the motor can be quickly responded to the requirements of the engine speed, so as to realize two power sources for different proportion of coordinated control of torque output.

\subsection{Motor Direct Torque Control}

Direct torque control (DTC) technology is put forward in the $1980 \mathrm{~s}$ of the high-performance control strategy of ac motor adopts robust control, the control principle of quick response, can achieve the fast response of the motor. Direct torque control in general is based on the mathematical model of asynchronous motor, speed control as the outer loop control, torque as the inner ring control, and strengthen the dynamic torque response of the torque performance. 
Hybrid test platform using Siemens MM440 frequency converter for motor control components, MM440 inverter control is divided into vector closed-loop control without speed encoder and vector closed-loop control of speed encoder, this article USES the belt speed encoder torque control of vector control, using current loop as feedback signal. Compared with vector control, the experiment of the feedback signal only current loop, rotational speed, in practice, only need to the maximum and minimum speed limit. Direct torque control, torque signal by P1503 setting, the control mode is determined by $\mathrm{P} 1300=23$, at the same time should be set according to the relevant data of the motor running parameters, the control system of dynamic and static optimization, so as to realize the optimization of motor control.

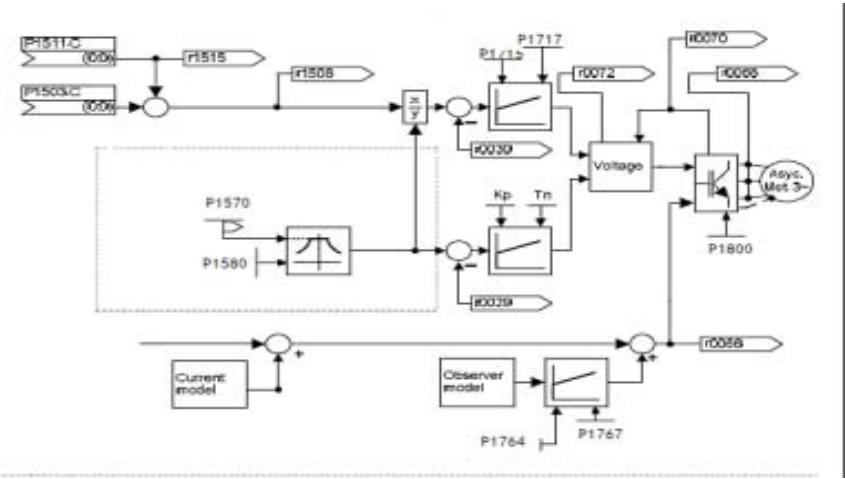

Figure 2. Inverter closed loop torque control block diagram

\section{Experimental Study}

\subsection{Bench consisting}

The experiment hybrid test bench device we used in this article is shown in figure 3, the output of the motor and engine through dynamic box output, load simulation by hydraulic system.

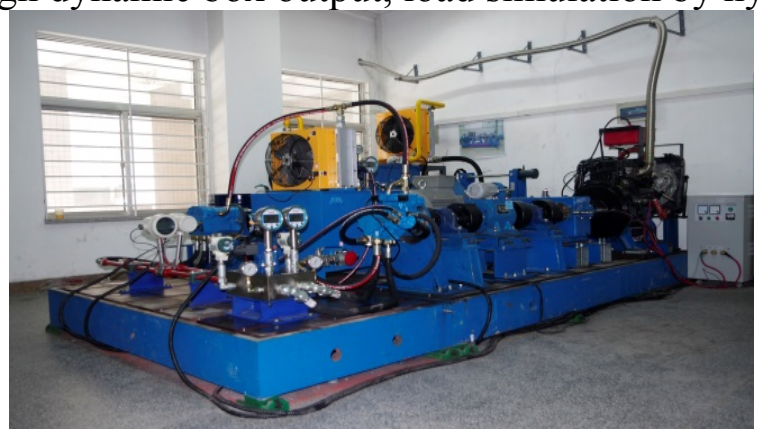

Figure 3. Hybrid test bench

\subsection{Experimental work}

According to the test method used in this experiment research on single mode switch to hybrid engine, the experiment, the diesel engine work within the scope of the stable, clutch 1 in the frequency converter set the torque of the motor size, start to start the motor when the speed of the motor to achieve stable, clutch 2 combination, will drive alone by the engine work status switch to hybrid engine and motor. Because the experiment is a mechanical connection between components, must exist in the process of energy transmission loss, in order to determine the transmission efficiency of diesel engine and electric motor, need to work on the above mentioned each model calibration, thus more accurate when calculating dynamic synthetic diesel engine and electric motor torque allocation rate.

Actual experiment motor torque by the parameters of the frequency converter r008 reads, and r008 calibration by DAC and by USS communication with PLC for communication, setting related parameters in PLC, thus they can obtain the actual motor instantaneous torque value.

Motor output torque through dynamic box coupling with the engine output torque, coupling after the synthesis of torque by installing in the speed of the output shaft torque measuring instrument measurement, the measured results through RS232 serial interface, by installing the software on the computer. Here to choose the torque speed measuring instrument for JCZ22 intelligent sensor. 
Separately by the engine work status switch to the state of the hybrid process engine, motor, in normal working state, the switch at the beginning, due to the constant torque of the motor, engine torque of time lag, when the engine speed is equal to the motor speed, the clutch combination, due to the instability of the engine working, coupled to the output torque will have large fluctuations, has a great influence to the stability of the output.

\subsection{The constant load test}

In order to verify the vehicle operating mode and power allocation, the use of independent research and development of a hybrid platform for experimental analysis, the use of direct torque control, set the torque of the motor to different values, under the working condition of hydraulic load system fixed load, the diesel engine starting, motor start for coupling output, figure 4 for the experimental measurement data processing results.

Can see from the picture in a fixed load condition, change motor set torque value, the output of the diesel engine torque change, which can realize in the case of fixed coupling output torque, motor and diesel engine power allocation problem.

Can see from the picture in a fixed load condition, change motor set torque value, the output torque of a diesel engine change subsequently. Change, according to the diesel engine speed regulation characteristic curve can know, diesel engine output torque change in motor speed change.

\subsection{Load Test}

In order to further verify the hybrid experiment platform of working mode and power distribution, hydraulic load changes in a certain range, the use of fuzzy control to realize the torque of the motor control, so as to realize the motor to the output of the hybrid diesel engine power compensation, achieve the stability of the diesel engine output.

Can be seen from the figure 5, the output of the hybrid increase according to certain rule, when the hydraulic load motor on diesel engine power compensation, ensure the diesel engine under the condition of load change the stability of the output torque speed In order to achieve in the case of fixed coupling output torque, motor and diesel engine power allocation problem.

\section{Conclusions}

The research, in view of the independent research and development of the hybrid test bench design, due to the diesel engine in the normal operation of the relevant operation parameters of dangerous sex, according to the pure diesel engine driven to hybrid mode switching parameters before and after the change, and the relationship between the parameters and working parameters of the diesel engine is obtained, and analyze the working condition of the diesel engine.

Bench test results show that the hybrid system by diesel engine switch to the diesel engine and electric motor drive system, the hybrid motor torque output torque and the known, based on the relationships between the mixing torque, be able to get the actual torque of the diesel engine, which can realize the analysis of operation condition of the diesel engine.

Because, in the process of clutch combination of instantaneous output system has certain impact, make the output of the hybrid system is not stable, how to take corresponding measures to optimize the impact, will be the content of further research.

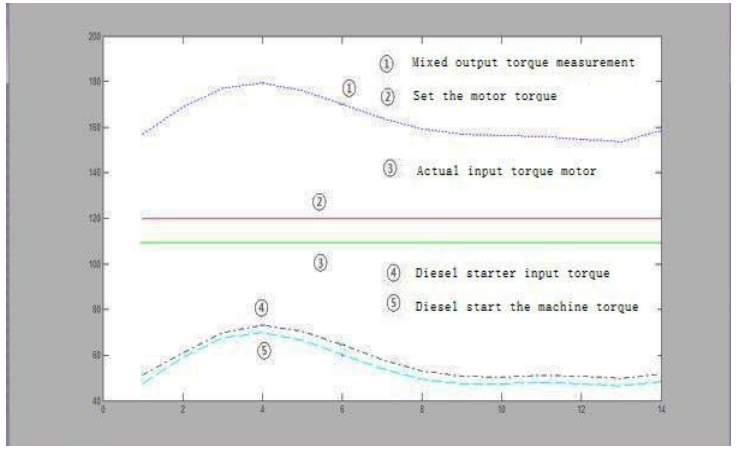

a

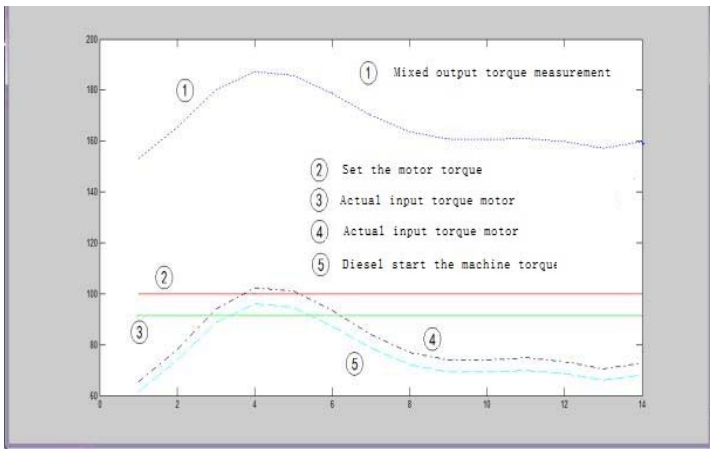

b

Figure 4. The results of the constant load test 


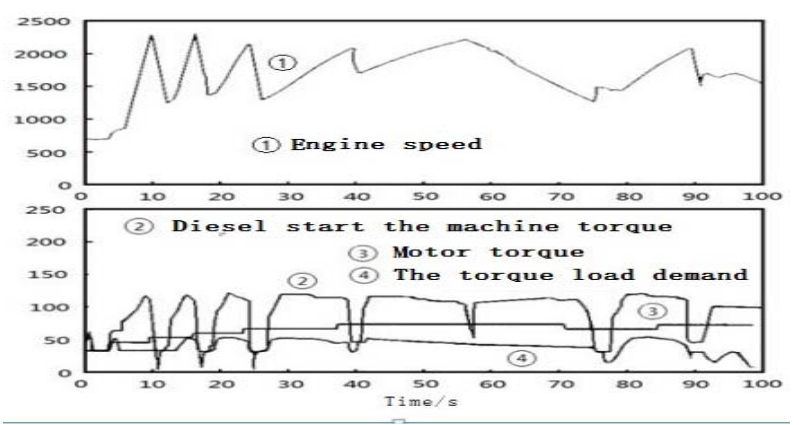

Figure 5. Load results

\section{References}

[1] Ding, Xiying. \& Zhao Xin. 2013. Direct torque control based on voltage space vector modulation of hybrid permanent magnet synchronous motor running at low speed performance improvement [J]. Electric technology, 4:27-41.

[2] Lin, C-C. \& Peng Huei.2003. Power management strategy for a parallel hybrid electric truck [J].IEEE Transaction on Control Systems Technology, 11(6):839-849.

[3] Ni, Chengqun. \& Zhang, Youtong.2013. Hybrid clutch combination process of dynamic torque control strategy [J]. Journal of mechanical engineering, 49 (4):114-121.

[4] Sun, Wenkai. 2007. Parallel hybrid electric vehicle system modeling and dynamic torque control simulation [M].

[5] Wang, Weihua. \& Jin, Qiqian.2005. Hybrid powertrain test bench study [J]. China journal of highway and transport, 18(2):103-106.

[6] Yin, Chengliang. \& Huan, PuJin. 2006. Parallel hybrid fuzzy torque control strategy [J]. Journal of Shanghai jiao tong university, 40(1):157-162.

[7] Yin, Chengliang. \& Pu, Jinhuan. 2006. Fuzzy torque control strategy of parallel hybrid [J]. Journal of Shanghai jiaotong university, 40 (1):157-162.

[8] Zhao, Zhi-guo. \&He, Ning. 2011. Four-wheel drive hybrid drive mode switch control [J]. Journal of mechanical engi neering, 47(4):100-109. 